Editor's Note: These short reviews of a recent paper in the Journal, written exclusively by graduate students or postdoctoral fellows, are intended to mimic the journal clubs that exist in your own departments or institutions. For more information on the format and purpose of the Journal Club, please see http://www.jneurosci.org/misc/ifa_features.shtml.

\title{
Transducin in Rod Photoreceptors: Translocated When Not Terminated
}

\author{
Deepak Kalra, ${ }^{1}$ Rebecca Elsaesser, ${ }^{2}$ Yi Gu, ${ }^{3}$ and Kartik Venkatachalam ${ }^{2}$ \\ ${ }^{1}$ Graduate Program in Biochemistry, Cellular, and Molecular Biology, ${ }^{2}$ Department of Biological Chemistry, and ${ }^{3}$ Graduate Program in Biological \\ Chemistry, The Johns Hopkins University School of Medicine, Baltimore, Maryland 21205 \\ Review of Lobanova et al. (http://www.jneurosci.org/cgi/content/full/27/5/1151)
}

Phototransduction in the vertebrate retina occurs in the outer segment (OS) of photoreceptor cells. Translocation of signaling proteins between the OS and the inner segment (IS) (Sokolov et al., 2002; Lee et al., 2003) allows these cells to adapt to a wide range of light intensities. This adaptation is evolutionarily conserved in vertebrates and invertebrates. Lobanova et al. (2007) in their recent study in The Journal of Neuroscience elucidate the mechanism for light-induced translocation of the G-protein transducin from the OS to the IS, indicating that saturation of the GTPase-activating complex (GAC) during high light intensities triggers translocation.

Transducin translocation in rod photoreceptors occurs when light intensity reaches a critical threshold (Sokolov et al., 2002). This threshold is reduced if the regulator of G-protein signaling 9 (RGS9), a component of the GAC, is knocked out or if a GTPase activity-deficient variant of the $\alpha$ subunit of transducin (Gt $\alpha$ ) is expressed (Kerov et al., 2005). The resulting increase in the activated form of the $\alpha$ subunit of transducin (Gt $\alpha-\mathrm{GTP}$ ) increases downstream signaling events.

Received March 28, 2007; revised April 30, 2007; accepted May 1, 2007.

We thank Drs. Craig Montell, Richard L. Huganir, and King-Wai Yau and apologize for any citations we left out because of space restrictions. None of us have any conflict of interest.

Correspondence should be addressed to Kartik Venkatachalam at the above address. E-mail: kvenkat2@jhmi.edu.

DOI:10.1523/JNEUROSCI.1399-07.2007

Copyright $\odot$ 2007 Society for Neuroscience $\quad$ 0270-6474/07/276349-03\$15.00/0
However, it is not clear whether increased levels of Gt $\alpha$-GTP are sufficient to trigger transducin translocation or whether downstream signaling events also play a role. To distinguish between these possibilities, Lobanova et al. (2007) compared light thresholds for transducin translocation in two mice models. RGS9-anchor protein (R9AP) knock-out mice lack the GAC and display physiological phenotypes similar to RGS9 knock-outs. The W70A mice have a mutation in transducin that decreases the affinity between phosphodiesterase and Gt $\alpha$-GTP, causing decreased downstream signaling. This mutation also abolishes RGS9-mediated increase in the GTPase activity of Gt $\alpha$ GTP. Therefore, after an increase in light intensity, both R9AP and W70A mice have increased levels of Gt $\alpha$-GTP, but only the R9AP mice have increased downstream signaling.

Compared with wild-type mice, the light threshold for transducin translocation in R9AP knock-out and W70 mice was reduced by $\sim 2.3$-fold [Lobanova et al. (2007), their Figs. 1 (http://www. jneurosci.org/cgi/content/full/27/5/1151/ F1), 2 (http://www.jneurosci.org/cgi/ content/full/27/5/1151/F2), 3 (http:// www.jneurosci.org/cgi/content/full/27/5/ 1151/F3)]. This result suggests that the threshold for transducin translocation decreases if Gt $\alpha$-GTP levels increase, regardless of the state of signaling events downstream of transducin. Overexpression of
R9AP (thereby increasing activity of the entire GAC) also increased the light threshold for transducin translocation [Lobanova et al. (2007), their Fig. 4 (http://www.jneurosci.org/cgi/content/ full/27/5/1151/F4)]. Because these perturbations increased Gt $\alpha$-GTP, a mouse model with defective signaling downstream of transducin but with normal RGS9-mediated increase in GTPase activity, (e.g., mutants with catalytically inactive phosphodiesterase) may enable a better understanding of the role of downstream signaling events under basal Gt $\alpha$-GTP levels.

The transducin $\beta$ - $\gamma$ dimer (Gt $\beta \gamma$ )binding protein, phosducin, is phosphorylated in the dark and dephosphorylated in light. In vitro, dephosphorylated phosducin binds Gt $\beta \boldsymbol{\gamma}$ with higher affinity than its phosphorylated counterpart (Thulin et al., 2001). This interaction prevents the association between Gt $\beta \gamma$ and Gt $\alpha$, thereby promoting their lightmediated translocation. The authors compared phosphorylation of phosducin in eye homogenates using phosphospecific antibodies and light intensities that allowed translocation in R9AP knock-out and W70A mice but not in wild-type animals. Phosducin dephosphorylation was complete in R9AP and wild-type mice but only partial in W70 mice [Lobanova et al. (2007), their Table 1 (http://www.jneurosci.org/cgi/content/ full/27/5/1151/T1)]. This strongly sug- 
gests that phosducin dephosphorylation is not sufficient for transducin translocation. A conclusive experiment would be to mutate the residues in phosducin that are phosphorylated and determine the effects on light threshold of translocation in vivo.

The transducin trimer consists of two posttranslationally added lipid anchors that tether it to membranes. Gt $\boldsymbol{\gamma}$ has a farnesyl group, whereas Gt $\alpha$ is modified on its $\mathrm{N}$ terminus with one of four different acyl groups. The authors purified Gt $\alpha$ from rat retinas and identified all four types of lipid moieties by mass spectrometry [Lobanova et al. (2007), Table 2 (http://www.jneurosci.org/cgi/content/ full/27/5/1151/T2)]. Using serial tangential sections of the retina, the authors analyzed both the distribution of transducin and the nature of the attached lipid moieties by Western blotting and mass spectrometry. This approach allowed the authors to directly compare the relationship between the nature of the lipid moiety attached to transducin and its distribution in the OS or IS. In dark-adapted animals, all four acylated isoforms of Gt $\alpha$ were distributed evenly in the OS. However, in rats exposed to light, transducin was redistributed along the entire length of the photoreceptor cell, including the IS [Lobanova et al. (2007), their Fig. 5 (http:// www.jneurosci.org/cgi/content/full/27/5/ $1151 / \mathrm{F} 5)]$. Interestingly, the different acylated isoforms of Gt $\alpha$ did not translocate to the same extent. The least lipophilic isoform was enriched in the IS by 1.5-fold, whereas the most lipophilic was depleted in the IS by fourfold. The results of this elegant experiment show that the lipophilicity of the acyl group attached to Gt $\alpha$ inversely affects its rate of translocation from the OS to the IS, most likely by increasing its affinity for photoreceptor membranes.

The transducin trimer is attached to the membrane by two lipid anchors. However, after dissociating from each other in light, Gt $\alpha$ and Gt $\beta \gamma$ are each attached to the membrane by single lipid anchors. These individual subunits have lower membrane affinity than the trimer (Seitz et al., 1999), thereby predicting a uniform increase in translocation with increasing light intensity rather than a critical light threshold. However, Lobanova et al. (2007) proposed a model [their Fig. 6 (http://www.jneurosci.org/cgi/content/ full/27/5/1151/F6)] (Fig. 1) whereby the threshold of light activation correlates directly with the capacity of the GAC to activate the intrinsic GTPase activity of Gt $\alpha$. Briefly, at light intensities below thresh-
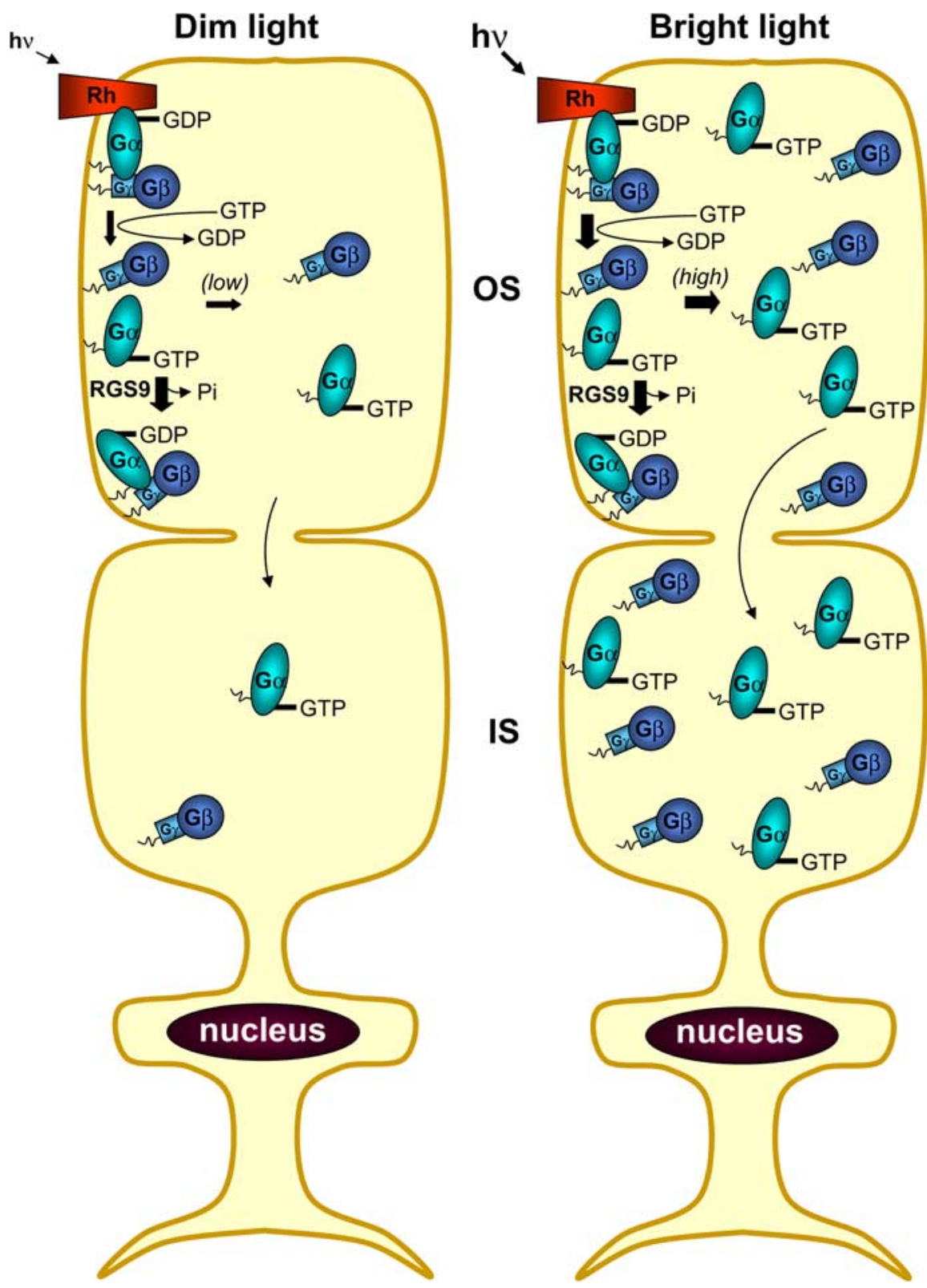

Figure 1. Light-dependent translocation of transducin. Left, In dim light, RGS9-induced inactivation of transducin prevents its rapid dissociation from the membrane. Thus, most of the transducin remains in the OS of the rod cells. Right, In bright light, higher levels of activated transducin overwhelm the RGS9 complex, thereby allowing the accumulation of activated transducin. The latter dissociates from the membrane and subsequently translocates to the rod IS. Rh, Rhodopsin; $\mathrm{h} \nu$, light; Pi, phosphate group.

old, GAC immediately inactivates Gt $\alpha$ GTP, thereby preventing its membrane dissociation. However, at light intensities above threshold, the capacity of the GAC to inactivate Gt $\alpha$-GTP is exceeded, so that transducin stays activated and dissociates from the membrane.

Despite this explanation, the function of transducin translocation remains enigmatic, because the light threshold of translocation is in the range at which rods saturate and cones take over vision. One intriguing explanation for this could be that the primary function of translocation is not to enable phototransduction at high light intensities but to prevent constant stimulation of rods when they are not contributing to vision. Persistent stimulation of all sensory neurons causes cell death. Indeed, excessive stimulation of the phototransduction cascade leads to retinal degeneration in vertebrate and invertebrate models (Yoon et al., 2000; Lee and Montell, 2004; Fain, 2006). Therefore, transducin translocation may have a valuable role in neuroprotection. Elucidation of the mechanisms involved in its translocation may assist in the development of therapeutic strategies for human retinal degenerations. 


\section{References}

Fain GL (2006) Why photoreceptors die (and why they don't). BioEssays 28:344-354.

Kerov V, Chen D, Moussaif M, Chen YJ, Chen CK, Artemyev NO (2005) Transducin activation state controls its light-dependent translocation in rod photoreceptors. J Biol Chem 280:41069-41076.

Lee SJ, Montell C (2004) Suppression of constant-light-induced blindness but not retinal degeneration by inhibition of the rhodopsin degradation pathway. Curr Biol 14:2076-2085.

Lee SJ, Xu H, Kang LW, Amzel LM, Montell C (2003) Light adaptation through phospho- inositide-regulated translocation of Drosophila visual arrestin. Neuron 39:121-132.

Lobanova ES, Finkelstein S, Song H, Tsang SH, Chen CK, Sokolov M, Skiba NP, Arshavsky VY (2007) Transducin translocation in rods is triggered by saturation of the GTPaseactivating complex. J Neurosci 27:1151-1160.

Seitz HR, Heck M, Hofmann KP, Alt T, Pellaud J, Seelig A (1999) Molecular determinants of the reversible membrane anchorage of the G-protein transducin. Biochemistry 38:7950-7960.

Sokolov M, Lyubarsky AL, Strissel KJ, Savchenko AB, Govardovskii VI, Pugh Jr EN, Arshavsky VY (2002) Massive light-driven translocation of transducin between the two major compartments of rod cells: a novel mechanism of light adaptation. Neuron 34:95-106.

Thulin CD, Savage JR, McLaughlin JN, Truscott SM, Old WM, Ahn NG, Resing KA, Hamm HE, Bitensky MW, Willardson BM (2001) Modulation of the $\mathrm{G}$ protein regulator phosducin by $\mathrm{Ca} 2+/$ calmodulin-dependent protein kinase II phosphorylation and 143-3 protein binding. J Biol Chem 276:23805-23815.

Yoon J, Ben-Ami HC, Hong YS, Park S, Strong LL, Bowman J, Geng C, Baek K, Minke B, Pak WL (2000) Novel mechanism of massive photoreceptor degeneration caused by mutations in the trp gene of Drosophila. J Neurosci 20:649-659. 